\title{
REVUE
}

\section{Arbres, arbustes, buissons et fourrages spontanés divers en régions tropicales et subtropicales (suite)}

\author{
par M.-G. CURASSON
}

\section{CHENOPODIACÉS (1) \\ COMBRÉTACEES}

\section{Genre Anageissus}

Ce genre a des représentants on Africue ot en Asie. Anageissus levicarpus et A. Schimperi sont des arbres de la savane de l'ouest africain, du Soudan Egyptien. A. latifolia, A. pendula sont des espèces de l'Inde. Toutes ont des feuilles comestibles.

\section{Genre Combretum}

Ce genre comprend de très nombreuses especes donf quelques-unes seulement sont fourageres. C'est le cas de Combretum aculeatum, de la région sahélienne, qui est recherchée des ruminants, surtout des chères; C. apiculatum, d'Afrique du Sud, qui est la seule espèce du genre considérée, dans cette région, comme intéressante; les feuilles et les pousses sont surtout mangées quand elles sont jeunes. De même celles de diverses espèces d'Afrique orientale, dont C. bonderianum, C. purpureifforum.

Plusieurs espèces d'Afrique sahélienne, du Soudan égyptien sont des fourrages médiocres. La composition des feuilles de $C$. apiculatum est la suivante : protéine brute, 12,6; cellulose brute, 28,5 .

\section{Genze Gujera}

Gujera senegalensis est un arbre de l'Afrique occidentale dont les feuilles sont distribuées aux vaches, en Guinée. Les Peuls leur attribuent des propriétés galactagogues et vont jusqu'à utiliser une infusion de ces feuilles pour laver les récipients dans lesquels est recueilli le lait, dans l'espoir d'augmenter la quantité de crèrne.

(1) Voir cette Revue 1051-1952, $\mathrm{z}^{\circ} \mathrm{I}$.

\section{Genre Terminalia}

Un arbre de la savane soudanaise, Terminalia avicenniotdes, a de grandes feuilles duveteuses ( $\alpha$ oreilles d'ane $w$ ) que mangent tous les riminants. De même T. macrophera. Dans l'inde, les bovins, moutons et chèvres mangent les feuilies de T. bellerica, de T. tomentosa. En Australie, celles de T. oblongata sont considérées comme excellentes.

\section{COMMRELUACEES \\ Genre Coramelina}

Commelina Forskalei est une herbe répandue dans les zones sablormeuses sèches d'Afrique occidentale; elle envahit parfois les champs d'arachides; c'est un bon fourrage pour tout le bétail ; on le vend parfois sur les marchés. C. nuciffora, du Guatemala, est également très appréciée.

\section{COMPOSEES \\ Genre Achillea}

Plusieurs espèces asiatiques du genre, des régions désertiques, sont mangées par le charneau, notamment Achillea falcata, A. santolina, A. vermicularis.

\section{Genre Ambrosia}

Ambrosia senegalensis est une petite Composée de la zore sahélienne que mange volontiers le mouton. A. maritima est une espèce de la zone soudanaise.

\section{Genre Amellus}

Amellus trigasus forme en Afrique du Sud des buissons qu'affectionne le mouton.

\section{Genze Anthemis}

Anthemis glareosa, "marglerite bâtarde * de Tripolitaine est fréquente dans la zone maritime. Elle est vendue sur les marchés et conservée sèche. 


\section{Genre Anvillea}

Plante saharienne et de Tripolitaine, Anvillea radiata est mangee par le mouton.

\section{Genre Argeratum}

Argeratum conizordes est une Composée annuelle qu'on trouve, parfois en assez grande étendue, en Afrique occidentale, en Afrique orientale, a Java. A Java, elle est mangée par le bétail; en Afrique occidentale, elle est considérée comme tokique pour les boeufs. En Ouganda, sa toxicité a été démontrée (Mettam).

\section{Genre Artemisia}

Il existe de très nombreuses espèces de ce genre dans les déserts et subdéserts africains et asiatiques. Artemisia armeniaca, $A$. arenaria, $A$. austriaca, A. campestris, A. dracunculus, A. ferganenis, A. Eragrans, A. herba alba, A. funcea, A. karataviensis, $A$. Jessingiana, $A$. leucodes, $A$. maritima, $A$. pauciIlora, A. persica, A. scoparia, A. scopariceformis, $A$. spinescens, $A$. sublessingiana, $A$. tianschanjca, A. turanica sont des especes du Proche-Orient, d'Asie centrale, d'U.R.S.S.; plusieurs espèces sudaméricaines sont mangées.

A. herba alba est retrouvée en Afrique du Nord, où on l'accuse de causer des accidents intestinaux; on y rencontre aussi $A$. campestris; $A$. variabilis existe en Tripolitaine.

Certaines espèces, telle $A$. taurica, peuvent être toxiques (particulièrement pour le mouton et le cheval); d'autres confèrent au lait un goût aromatiquo, telle $A$. verlotorum (Argentine).

La composition d' $\dot{A}$. herba alba, en fleurs, est la suivante : proténe brute, 6,4 ; extrait éthéré, 5,1 ; cellulose brute, 38,4 ; extractif non azoté, 43,8 ; cendres, 6,3 ; celle d'A. maritima, en fin de végétation, est ainsi : protéine brute, $9_{x} 2$; extrait éthéré, 8,6; cellulose brute, 29,1; extractif non azoté, 43.

\section{Gense Aster}

De bonnes plantes de ce genre constituent, avec d'autres Composées, la partie la plus importante des buissons fourragers du Karoo (Afrique du Sud). Less principales espèces sont Aster barbatus, $A$. filfolius, $A$. muricatus, $A$. muricatus var. fascicularis. A. filifolius est accusée de toxacitê (Steyn, 1934) raais cela ne parait pas correspondre à la pratique (Henrici, 1944). La composition d'A. filifolius (en période de sécheresse) est la suivante : eau, 52,7; protéine brute, 10,7 ; extrait éthéré, 6,8 ; cellulose brute, 18,1; extractif non azoté, 55,3; cendres, 9,1.

\section{Genre Atractylis}

Atractylis cancellata, A. flava, d'Afrique du Nord, A. serrutaloides, de Libye, Tripolitaine, sont surtout mangées par le chameau.

\section{Genre Baccharis}

Les espèces de ce genre jouent un certain rôle dans les pâturages d'Amérique. Baccharis artemisioides, du Brésil, que le bétail mange en période de disette, et qui donne une odeur désagréable au lait; B. tenuifolia; B. conferta (Mexique), B. coridifolia, en Argentine, est toxique, et on recommande son éradication; le betail indigène la délaisse ordinairement, mais les anmaux imfortés peuvent la manger. Un curieux procédé consiste à enfumer les animaux avec la plante brûlée, pour provoquer chez eux de la répugnance. $B$. magellanica, d'Argentine, $B$. trimera, d'Uruguay, sont surtout mangées par les moutons. $B$. halimifolja est une espèce d'Ámérique du Nord.

\section{Genre Brachyglottis}

Brachygiottis repanda est un arbre ou arbuste de Nouvelle-Zélande dont les très grandes feuilles sont mangées par le bétail. Elles pourraient être dangereuses.

\section{Genre Calendula}

Calendula officinalis, mangée par le chameau et les moutons, est récoltée et vendue sur les marchés, en Libye, en Tripolitaine.

\section{Genre Carduncellus .}

Carduncellus acaulis est mangée par le chameau en Tripolitaine. On la récolte aussi pour les bovins.

\section{Genre Centaurea}

Sont mangées : en Afrique occidentale (Nigeria), Centaurea calcitrapa; en Libye, C. dimorpha, qui est vendue sur les marchés. C. contracta est également mangée, mais serait dangereuse en grande quantité. C. senegalensis est une espèce de peu de valeur qui pousse sur les dunes de la zone sabélienne, comme C. alexandrina, C. praecox.

\section{Genre CJadanthus}

Cladanthus arabicus, d'Afrique du Nord, mangée par le chameau, pourrait être dangereuse.

\section{Genre Cynara}

Cynara cardunculus, d'Afrique du Nord, est une bonne espece pour lo chameau seulement. 


\section{Genre Drosanthemum}

Drosenthemum intermediun est bien appétée des animaux en Afrique du Sud.

\section{Genre Eclipla}

Eclipla alba est une Composée du bord des mares sahéliennes que mange volontiers le mouton.

\section{Genxe Elythropappus}

Des buissons d'Afrique du Sud appartenant à ce genre ont une valeur fourragère parfois nulle ou faible, tel Elythropappus rhinocerotis qui devient parfois une plante envahissante, ou meilleure (E. gnaphaloides).

\section{Gente Enchylcena}

Enchylona tomentosa (sous ce nom sont probablement réunies plusieurs especces) est une Composée arbustive australienne qui est considérée comme un bon fourrage.

\section{Genre Eriocephalus}

Des plantes de ce genre, assez résistanfes à la sécheresse, constituent en Afrique du Sud des fourrages de valeur moyenne : Erjocephalus glaber, $E$. exicoides, E. spinescens, E. umbellatus, E, racemosus. Elles sont en général recherchées.

La composition d'E. glaber est la suivante (saison sèche) : eau, 46,3; protéine brute, 10; extrait éthéré, 5,2 ; cellulose brute, 26,8; extractif non azoté, 49,8; cendres, 8,3.

\section{Genre Earyops}

Des plantes buissonneuses de ce genre sont, en Afrique du Sud, des fourrages médiocres ou moyens; certaines sont très amères. On peut retenir : $E$. asparagoldes, E. athanasive, E. laterifolius, E. multifidus. Ia composition de cette dernière est la suivante : eau, 73,5 ; protéine brute, 17 ; extrait éthéré, 4,1 ; cellulose brute, 15,2 ; extractif non azoté, 45 ; cendres, 13,5 .

\section{Genre Fradinia}

Fradinia halimifolia, d'Afrique du Nord, est mangèe par le chameau, mais pourrait être dangereuse.

\section{Genre Francæuria}

Francaeuria crispa forme dans le Sahara un arbrisseau que mangent surtout les chameaux.

\section{Genre Geijera}

Une Composee arbustive australienne, Geijera parviflora, est une bonne plante à moutons, qu'on peut tailler et qui est bien appétée. G. Salicifolia est également mangée. La composition de G. parviflora est la suivante : protéine brute, 14; extrait éthéré, 4 ; cellulose brute, 12,8; extractif non azoté, 60,6; cendres, 9,4 .

\section{Genre Helianthus}

Le soleil commun, ou tournesol, Helianthus annuus est surtout cultivé en régions tempérées ou tempérées chaudes, pour sa graine ou pour la plante entière quáon donne en vert ou ensilée. En régrions chaudes, on peut profiter de la saison des pluies ou recourir à l'irrigation. Quand la saison des pluies est courte, on est obligé de couper en vert, les graines ne pouvant venir à maturité. Il est fréquent, de toute facon, oqu'une forte proportion de graines avortent.

\section{Genre Helichrysum}

De'bons fourrages sont constitués par plusieurs Helichrysum d'Afrique du Sud; d'autres sont indifférentes et même nocives. Les plus importantes sont $H$. luciliojdes, $H$. dregeanum, H. Zeyheri, $H$. parviflorum. H. pentzoiddes. Certaines espèces ne pourraient pas être mangées seules, mais devraient être mélangées à des fourrages plus pauvres.

Certaines sont dangereuses parce que vulnérantes. La composition d'H. pentzoldes est la suivante : eau, 61,2; protéine brute, 8 ; extrait éthére, 2,2 ; cellulose brute, 23,8 ; extractif non azoté, 41,2 ; cendres, 24,7 .

\section{Genxe Hertia}

Hertia ciliata, H. cluytiæfolia, H. pallens sont de bonnes plantes à mouton (Afrique du Sud). H. pallens est considérée comme toxique.

\section{Genre Horpicium}

Horpicium echinulatum, $H$. integrifohum sont des Composées bussonneuses d'Afrique du Sud, assez répandues et bonnes.

\section{Genre Inula}

Inula ammophila, d'Afrique du Nord, est mangée par le chameau.

\section{Genre Lactuca}

Lactuca taraxacifolia, la laitue sauvage du Sénégal (" langue de vache ") est distribuée aux chèvres, aux brebis, aux vaches. Elle est considérée comme galactagogue et comme favorisant les naissances multiples par les Peuls du Niger, de Nigeria.

\section{Genre Metalasia}

Deux Composées arbustives d'Afrique du Sud, Melalasia bolusii, $M$. muricata, sont mangées surtout par le mouton. 


\section{Genre Nestlera}

Des buissons d'Afrique du Sud, Nestlera congesta, $N$. humilis, $N$. conferta, sont mangés volontiers.

\section{Genre Nolletia}

Nolletia chrysocomoldes, d'Afrique du Nord, est mangée par le chameau.

\section{Genre Osteospermum}

Des Composées de ce genre, Osteospermum moniliferum (avec les sous-variétés lanosum et angustifolium), 0 . muricalum, sont répandues et recherchées en certaines régions d'Afrique du Sud. La dernière espèce se rencontre aussi en Rhodésie. Elle renferme, transformée en foin, $12,1 \%$ de protéine, $4,3 \%$ de matières grasses et $42,5 \%$ d'hydrates de carbone.

\section{Genre Othonna}

Othona pteronioĩdes, O. triplinervia, d'Afrique du Sud, sont de valeur moyenne.

\section{Genre Pegolettia}

Un buisson xérophile à feuilles succulentes ou non, selon le terrain et la pluviométrie, constitue, en Afrique du Sud, un bon fourrage : Pegolettia retrofracta. $P$. polygalælolia est également résistant à la sécheresse et a de bonnes feuilles. Sa composition est la suivante : eau, 65,2 ; protéine brute, 14,6 ; extrait éthéré, 7,2 ; cellulose brute, 20,$4 ;$ extractif non azoté, 48,9 ; cendres, 9 .

\section{Genre Perralderia}

Perralderia coronœifolia, d'Afrique du Nord, est mangée par le chameau, mais pourrait être dangereuse.

\section{Genre Phymaspermum}

Phymaspermum parviflorum est parmi les buissons les plus répandus du Karoo; sa composition vaut celle d'une bonne herbe et cependant le coefficient de digestibilité est faible. L'analyse des feuilles donne les résultats suivants : eau, 65,1; protéine brute, 14,8; extrait éthéré, 3,4; cellulose brute, 24,6 ; extractif non azoté, 48,5 ; cendres 12,2 .

\section{Genre Plazia}

Plazia argentea est une Composée d'Amérique du Sud dont la multiplication est recommandée pour la fixation des dunes; elle est comestible.

\section{Genre Pteronia}

Des espèces assez nombreuses de ce genre existent en Afrique du Sud. Certaines sont fourra- gères, d'autres indifférentes et même toxiques. Ainsi, Pteronia punctata, Pt. glaucescen, Pt, erythrochaeta sont mangées, alors que $P$ t. tricephala ne l'est pas, et que Pt. pallens est toxique.

\section{Genre Pyrethrum}

Plusieurs espèces du genre, dans les déserts asiatiques ou le Sahara, sont mangées par le charneau et le mouton.

\section{Genre Relhanja}

Relhania cuneata, R. genistoefolla, R. squarrosa sont des Composées buissonneuses, très répandues en certaines régions d'Afrique du Sud. Ce sont surtout les fleurs qui sont mangées.

\section{Genre Rhanteritum}

Rhanterium adpressum et $R$. suaveolens constituent des buigsons du Sahara que mange le chameau.

\section{Genre Santolina}

Santolina squammosa forme dans le Sahara des buissons mangés par le chameau.

\section{Genre Scabiosa}

Scabiosa camelorum, en Afrique du Nord, est recherchée par le chameau.

\section{Genre Senecio}

On sait que, parmi les espèces tropicales de ce genre, beaucoup se montrent toxiques. Certanes, cependant, sont mangées sans que soient signalés des accidents : $S$. Greyii, de Nouvelle-Zélande; $S$. reptans, d'Afrique du Sud; $S$. bonariensis, d'Uriguay; $S$. mendocinus, d'Argentine.

\section{Genre Stcebe}

Stcobe capitata est une Composée buissonneuse mangée en Afrique du Sud.

\section{Genre Tarchonanthrs}

Tarchonanthus camphoratus, T. minor forment dans le Karoo des buissons qu'affectionne le mouton. La composition de T. minor est la suivante : eau, 51,3; protéine brute, 12,1 ; extrait éthéré, 11,6 ; cellulose brute, 25,6; extractif non azoté, 44 ; cendres, $6,7^{\circ}$

\section{Genre Tripteris}

Ce genre est représenté en Afrique dư Sud par plusieurs espèces intéressantes. La meilleure est Tripteris pachypteris, très répandue en certains 
endroits; elle est remplacée par Tr. spinescens dans les régions arides du Karoo. Tr. leptoloba est également une bonne espèce. Ces buissons pelivent suffire à eux seuls pendant un certain temps à l'alimentation du mouton. Cependant la richesse de Tr. pachypteris en sulfate de chaux peut être $\mathrm{m}$ inconvénient.

\section{Genre Zollikoferia}

Plusieurs espèces du genre sont margées par le chameau en Afrique du Nord : Zollikoferia arabica, $Z$. microryncha, $Z$. nudicaulis, $Z$. resedifolia.

\section{CONIFÈres}

\section{Genre Juniperss}

Les pousses de Juniperus macrocarpa sont mangées par les chevres et les chameaux en Tripolitaine. Plusieurs espèces améncaines sont mangées : $J$. mexicana, $J$. pinchotti, $J$. virginiana. Dans l'Inde (Baluchistan) on distribue au chameau les baies de Juniperus.

\section{CONNARACÉES}

\section{Genre Cnestis}

Cnestis grisea est un arbrisseau d' frique orientale que mangent les petits ruminants; on peut observer des accidents, dus probablement aux poils urticants.

\section{Genre Santalum}

Santalum album, arbre de l'Inde, a des feuilles considérées comme bon fourrage. De même $S$. lanceolatum, du Queensland, d'Australie occidentale.

\section{CONVOLVULACEES}

\section{Genre Convolvalus}

Dans les terrains sablonneux mais humides, au Sahara, Convolvulus fatmensis est une bonne plante à chameau. De même C. Supinus, en Tripolitaine. Dans l'Inde, $C$. lejocalycinus, $C$. spinosus sont mangées par les divers herbivores.

\section{Genre Cressa}

Cressa cretica est une plante des terrauns salés du Sahara, de l'Inde, que mange volontiers le chameau.

\section{Genre Ipomce}

C'est à ce genre qu'appartient la patate cultivée, Ipomce batatas. Le tubercule n'est pas la seule partie utile. Les feuilles sont en effet un bon fourrage vert.
Leur composition, qui indique celle des Iponce en général est la suivante :

\begin{tabular}{|c|c|}
\hline & 85,4 \\
\hline Matières & 2,0 \\
\hline Matières & 0,56 \\
\hline Matières & 8,1 \\
\hline Matièr & 1,2 \\
\hline & \\
\hline
\end{tabular}

Dans les dépressions, au bord des mares et des cours d'eal, en régions désertiques, des lpomce peuvent être une ressource en vert. C'est le cas pour I. cardiosepala, du Soudan égyptien, bon pâturage à chameau après les pluies, mais purgatif au début; 1. repens, de la zone sahelienne est également mangée. mais parfois accusée d'être toxique; dans la même zone, existent $I$. coccinosperma, $I$. hispida, I. pestigridis, I. hallebarda, I. verticillata. Cette demière est très recherchée du bétail et n'est broutée qu'au début de la saison des pluies. Les nomades la considérent comme galactogène.

D'autres espèces peuvent être mangées : J. Wightii, d'Afrique orientale, peut être donné au mouton en alimentation exclusive sans causer d'accidents; $I$, reptans est mangée par l'homme en Indochine. On le trouve aussi en zone guinéenne.

Diverses espèces sont consommées en Amérique du Sud : 1. arborescens var. glabra dont les corolles tombées à terre sont mangées (Mexique); l. selifera (feuilles) au Brésil ; I. hederacea, I. pentaphylla, etc. , au Guatemala.

\section{Genre Merremia}

Merremia pedata est un fourrage pour chameau, en Sornalie, au Soudan égyptien. $M$. pentaphylla est une espèce de la zone soudanaise.

\section{CORTHRIMCEES}

\section{Genre Coriaria}

Plusieurs espèces du genre, en Nouvelle-Zélande, ont des feuilles mangées : $C$. arborea, C. pottsiana, C. sarmentosa sont les principales. D'autres espèces sont toxiques. De même C. rusufolia, d'Amérique du Sud.

\section{CORNACEES}

\section{Genre Coraus}

Cornus nuttallii, de la région côtière de Colombie britannique, est un arbre dont les feuilles sont mangées volontiers; de même celles d'espèces américaines : C. paniculata, C. stolonifera.

\section{Genre Geniostoma}

Les feuilles de Geniostoma litoralis, de NouvelleZélande, bien que coriaces, sont mangées par le 
bétail. On les distribue parfois. De même celles de G. Iucida.

\section{Genre Griselinia}

Griselinja litorales est parmi les espèces dont on conseille la propagation en Nouvelle-Zélande.

\section{CORYNOCARPaCÉES}

\section{Genre Corynocarpus}

Corynocarpa lœvigata est un grand arbre de Nouvelle-Zélande dont les longues feuilles sont recherchées du bétail. Elles sont parfois distribuées. Le fruit serait toxique.

\section{CRASSULACEES}

\section{Genre Kalanchce}

Plusieurs espèces de ce genre pourraient être mangées par le bétail, qui aime les feuilles succulentes; mais il est des espèces réputées toxiques.

\section{CRUCIFÈRES}

\section{Genre Brachycarpa}

Dans certaines régions montagneuses d'Afrique du Sud, Brachycarpa sp, est mangée par les moutons.

\section{Genre Brassica}

Brassica campestris est considérée comme bon fourrage vert dans I'Inde, pour le chameau notamment.

Plusieurs espèces du genre sont cultivées dans l'Inde. Brassica napus, dans le Punjab, pousse sous irrigation ou non. On le donne en vert et les graines, après extraction de l'huile, donnent un tourteau qu'on distribue aux animaux ou qu'on utilise comme engrais.

B. Oleracea (chou moellier) aime les climats tenipérés et l'humidité, mais cerlaines vanétés assez résistantes à la sécheresse peuvent être cultivées en régions subtropicales. On l'utilise comme fourrage vert.

'B. rutabaga est aussi cantonné à peu près exclusivement aux régions tempérées. Certaines variétés peuvent pousser en régions chaudes, sous irrigation. Nous l'avons vu réussir dans ces conditions au Soudan français.

\section{Genre Carthamus}

Carthamus tinctorius est cultivé dans l'Inde où, après extraction de l'huile, les graines fournissent un bon tourteau. On ensile aussi la plante entière. Celle-ci préfère les sols sablonneux.
C. oxyacantha, de l'Inde également, fournit un fourrage qui a la valeur alimentaire des pailles de céréales.

\section{Genre Eruca}

A côté de la roquette cultivée, Eruca sativa, qui est surtout utilisée pour sa graine, existe une espèce différente, $E$. pinnatifolia, la roquette fourragère, roquette des oasis, qui est entretenue dans les oasis sahariens. Elle pousse en trois mois, et on peut faire plusieurs semis.

\section{Genre Diplotaxis}

Diplotaxis pendula est mangée dans le Sahara.

\section{Genre Farsetia}

Farsetia sp., dans le sud saharien, est un bon pâturage à chameau.

\section{Genre Heliophila}

Les espèces sud-africaines, Heliophila suavissima, $H$. sulcata var. modestia, $H$. abrotanifolia forment des buissons qui sont broutés.

\section{Genre Henophyton}

Henophyton deserti, du Sahara, est un arbrisseau recherché du chameau.

\section{Genre Malcomia}

Malcomia aegyptiaca est mangée dans le Sahara.

\section{Genre Malthiola}

Malthiola Jivida, d'Afrique du Nord, est très répandue et recherchée après les pluies par'le chameau.

\section{Genre Montinea}

Montinea caryophyllacea, d'Afrique du Sud, est mangée par le mouton.

\section{Genre Morettia}

Morettia canescens du sud saharien est excellente pour tous les herbivores.

\section{Genre Moricandia}

Moricandia subfruticosa, d'Afrique du Nord, est mangé par le chameau.

\section{Genre Schouwia}

Schouwja arabjca, du sud saharien, est mangée par le chameau. 


\section{Genre Zilla}

Zilla macroptera, du Sahara, est un arbrisseau épineux très prisé du chameau.

\section{CUCURBITACEES \\ Genre Acanthosicyes}

Acanthosicyes horrida est une Cucurbitacée qui croit dans l'Angola et d'autres régions d'Afrique occidentale; épineuse, sans feuilles, elle est extrémement résistante à la sécheresse. Les fruits, sucrés, constituent une bonne alimentation.

\section{Genre Cephalandra}

Les fruits de Cephalandra indica, C. quingueloba, de la région sahélienne, sont mangés par les bovins, les chameaux. Une espèce voisine, des régions plus humides de l'Afrique occidentale. C. sylvatica, est toxique.

\section{Genre Cucumis}

Plusieurs espèces de Cucumis, qui poussent en terrains sablonneux, sont mangées par les animaux; leurs fruits sont recherchés malgré leur saveur parfois amère, en raison de leur teneur en eau (C. prophaterum). Les feuilles de la coloquinte, C. colocynthis, sont mangées. Plusieurs Cucunnis sont toxiques.

\section{Cyathé́acéES}

Une fougère arborescente de Nouvelle-Zélande, Crathea dealba, et une espèce plus petite, C. medullaris, ont des feuilles comestibles. Le tronc de Cibotium chamossii, fougere arborescente d'Hawaï, qui contient $3,7 \%$ de protéine et $82,1 \%$ d'hydrates de carbone, est également mangé.

\section{CYPÉRACÉES}

\section{Genre Bulbostylis}

Plusieurs espèces de ce genre sont des herbes poussant sur les dunes sahéliennes; elles sont mangées par les moutons.

\section{Genre Cyperus}

De nombreuses espèces du genre poussent, dans les diverses régions chaudes du monde, sur les bords sablonneux des cours d'eau, des mares, dans les champs, parfois sur les dunes; souvent elles ont des tubercules comestibles. Au bord des mares du Sahel, on trouve $C$. bulbosus, $C$. esculentus, $C$. macuJatus, C. articulatus; sur les dunes, C. jria, C. conglomeratus. Dans les terrains cultivés d'Afrique occidentale, $C$. rotundus est un fourrage moyen. Plus au sud, on trouve C. procerus. En Indochine, C. haspan, C. rotundus existent dans les rizières. Au Mexique, C. Bourgaei, C. sesleroides abondent dans les zones chaudes et humides.

\section{Genre Fimbristylis}

Fimbristylis diphylla, $F$. dichotoma, F. exilis, F. millacea se rencontrent sur les bords du Niger, parfois en gazon continu.

\section{Genre Juncellus}

Juncellus loevigatus et J. pygmeus poussent aux bords des mares sahéliennes.

\section{Genre Killinga}

Killinga monocephala tient une place parfois importante parmi les herbes de rizières, en Indochine.

\section{Genre Pycreus}

Pycreus polystachius est parmi les herbes de rizières dustribuées aux animaux en Indochine. $P$. tremulus forme parfois des gazons importants sur les bords du Niger.

\section{Genre Scirpus}

Scirpius erectus, d'Indochine (rizières), S. occultus, des bords du Niger, sont mangées par les bouns, les moutons.

Ces diverses Cyperacées forment souvent, au retrait des eaux, de jeunes herbes relativement tendres dont les pasteurs guettent l'appantion. Malheureusement elles sont souillées de terre, d'ceufs de parasites et assurent l'infestation périodique des animaux. Leur composition, concernant des espèces d'Indochine, est la suivante pour 100 (Remond et Winter, 1944).

\begin{tabular}{|c|c|c|c|c|}
\hline & $\begin{array}{l}\text { MATTERES } \\
\text { azatées }\end{array}$ & GRAISSES & $\begin{array}{l}\text { MATTieres } \\
\text { sèches }\end{array}$ & $\begin{array}{l}\text { Nombre de kilogrammes } \\
\text { correspondant à } 1 \text { unité fourragère }\end{array}$ \\
\hline Killinga monocephala .... & 11 & 0,8 & 150 & 7,5 \\
\hline Scirpus erectus. ..... & 9 & 0,5 & 150 & 8,5 \\
\hline Cyperus haspan........ & 12 & 0,7 & 150 & 7,5 \\
\hline 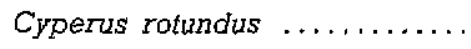 & 10 & 0,8 & 150 & 3,5 \\
\hline Pycreus polystachius........... & 8 & 0,7 & 150 & 9 \\
\hline
\end{tabular}




\section{EBENACÉES}

\section{Genre Diospyros}

Des arbustes de ce genre sont intéressants par leurs feuilles et leurs fruits. Diospyros mespiliformis, d'Afrique occidentale, foumit une grosse quantité de fruits sucrés que les moutons mangent avidement, pour régurgiter ensuite les noyaux. Plusieurs espèces du Texas, D. virginiana, D. texana, notamment, ont aussi des feuilles et fruits comestibles. Les fruits de $D$. virginiana ont la composition stivante : protéine brute, 3,3 ; extrait éthéré, 2,9; cellulose brute, 5,3; extractif non azoté, 87,4; cendres, 3,2 .

\section{Genre Euclea}

En Afrique du Sud, des arbres de ce genre sont considérés comme fourragers : Euclea natalensis, $E$. ovata, $E$. undulata, $E$. verrucosa. Très répandus, ils sont utiles quand il n'y a pas d'autre alimentation verte.

\section{Genre Royena}

On considère comme arbre fourrager, en Afrique du Sud, Royena pallens, dont les feuilles sont surtout mangées quand elles sont tombées, en hiver. Leur composition est la suivante : protéine brute, 7,4 i extrait éthéré, 9,9 ; cellulose brute, 24,2 ; extractif non azoté, 48 ; cendres, 10,2 .

\section{ELIEOCARPÉES}

\section{Genre Elaeocarprs}

Un grand arbre de Nouveile-Zélande, Elaeocarpus dentatus, foumit des graines avec lesquelles on engraisse les porcs.

\section{Genre Arristotelia}

Des espèces des forêts de Nouvelle-Zélande ont quelques valeurs : les fruits d"Aristotelia racemosa sont mangés par le bétail. De même pour $A$. macqui, d'Argentine.

\section{EUPHORBLACEES}

\section{Genre Acaljpha}

Ce genre renferme de nombreuses espèces des diverses régions chaudes. Certaines sont toxiques, d'autres douteuses, alors que des espèces sont mangées par le bétail, comme $A$. fructicosa, A. omata, d'Afrique orientale; $A$. punctata, d'Afrique du Sud.

\section{Genre Bridelia}

Bridelia retusa, de l'Inde, est considérée cornme de bonne qualité. Plusieurs espèces d'Afrique occi- dentale, également arbustives, pourraient être mangées ( $B$. ferruginea).

\section{Genre Chrozophora}

Chrozophora senegalensis, du Sahel, est bien appétée du chameau et du mouton, ainsi que Ch. brocchiana.

\section{Genre Euphorbia}

Diverses espèces de ce genre sont mangées, soit parce que leur suc n'est pas irritant, soit que les feuilles sont consommées sèches, tombées à terre, n'ayant plus de suc.

A Madagascar, le mouton s'attaque volontiers aux pousses et aux feuilles d'E. stenoclada.

En Afrique du Sud, plusieurs espèces sont d'assez bons fourrages : $E$. aspericaulis, $E$. Bergeri, $E$. caterviflora, $E$. ccerulescens, $E$. hamata, $E$. rhombifolia, $E$. stolonifera, $E$. truncata, $E$. esculenta, $E$. Burmannii, E. Turicalli.

Au Soudan égyptien, les chèvres et les chameaux mangent $E$. abyssinica.

Dans les déserts d'Asie centrale, E. gerardiana est mangée: sa composition est la suivante : protéine brute, 13,6; extrait éthéré, 5,4; cellulose brute, 21,1; extractif non azoté, 52,1 ; cendres, 7,2 .

En Afrique occidentale, les feuilles d'E. bajsamifera sont mangées quand elles sont tombées à terre. Plusieurs autres especes sont utilisées comme galactagogues, probablement en raison de l'aspect laiteux de leur suc; les feuilles sont mises à macérer dans l'eau de boisson : E. polycnemoides, E. convolvuloìdes, $E$. hirta.

Au Guatemala, E. lancifolia est considérée comme particulièrement bonne pour les vaches laitières.

\section{FICOIDEES}

\section{Genre Reaumuria}

Reaumuria sangarica, de l'Asie centrale, $R$. verticillata, du Sahara, sont des plantes à chameau peu importantes.

\section{Genre Sesuvium}

Sesu vium portulacastrum est une plante saharienne (Mauritanie) recherchée du chameau.

\section{Genxe Trianthema}

Trianthema pentendra, du Sahel, est aimé du chameau.

\section{FHXCOURTIACEES}

\section{Genre Oncoba}

Oncoba echinata est un arbre de la zone guinéenne d'Afrique occidentale dont les graines peurent être 
mangées; elles renferment $18 \%$ de proténe, $46 \%$ de graisse, i2 \% d'hydrate de carbone.

\section{FRANKÉNLACÉES}

\section{Genre Frankenia}

Frankenia lovis, arbuste de Tripolitaine, qui pousse en bordure des terrains salés, est mangé par le chameau. Plusieurs espèces du genre sont mangées au Chili par le mouton et la chévre.

\section{GERANIACEES}

\section{Genre Erodium}

Erodium arborescens, d'Afrique du Nord, de Tripolitaine est mangée par les chèvres et les chameaux.

\section{Genre Pelargonium}

Plusieurs espèces sud-africaines sont mangées, notamment, $P$. crithmifolitum, $P$. incisum.

\section{GESNERIACEES}

\section{Genre Koberia}

Koberia phleoldes, du Sahara, est appréciée de tous les herbivores.

\section{GLOBULARIACEes}

\section{Genre Globularia}

Globularia alypum est recherchée des chevres, en Lybie, Tripolitaine, ainsi que Gl. arabica.

\section{GNETRCÉS}

\section{Genre Ephedra}

Plusieurs especes du genre sont mangées par le chameau, la chèvre dans le Bahuchistan. En Tripolitaine, les fruits d'E. alatz sont mangés par le chameau, E. strobiculata, d'Asie centrale, E. nevadensis, du Texas, E. ochreata, E. tweediana, d'Ámérique du Sud, sont également mangées.

\section{ICRCINACEES}

\section{Genre Pozaqueiba}

Poraqueiba sericea est un arbre des zones arides tu Bresil, de bonne valeur fourragère.

\section{LEBIÉES}

\section{Genre Lasiocorys}

Lasiocorys capensis est un bon buisson fourrager lu Karoo (Afrique du Sud).

\section{Genre Lavandrla}

Lavandula coronopifolia et $L$. multifida, sont mangées en Tripolitaine. La première est dénommée "fourrage des ânes "; la seconde est recherchée du chameau. Diverses Luvandula existent dans les maquis méditerranéens.

\section{Genre Marrubjum}

Marrubium deserti est mangée par le chameau en Afrique du Nord.

\section{Genre Ocimum}

Les moutons et chèvres mangent volontiers des herbes de ce genre: Ocimum viride, 0 . amerjcanum, de la région sahélienne de l'Afrique, $O$. Sellor, d'Amérique du Sud.

\section{Genre Salria}

Plusieurs espèces du genre sont mangées en Afrique du Sud. S. nivea est la seule espèce arbustive.

\section{LAURACEES}

\section{Geure Beilschmiedia}

De petits arbres australiens, Beilschmiedia tawa et $B$. tarairi, ont leurs feuilles mangées par le bétail. On les considère parfois cormme suspects.

\section{LÉCYTHIDACEES}

\section{Genre Lecythis}

Lecythis pisonis est un arhre des régions arides du Brésil qui a une bonne valeur fourragère.

\section{LÉGOMDNEUSES}

Des trois sous-familles qui composent les Légumineuses, la plus importante, de notre point de vue, est celle des Mimosées qui comprend de nombreuses espèces fourragères. Les Mirnosées arborescentes constituent, en effet, par leurs feuilles tant que par leurs gousses, une nourriture très alibile, très riche en matière azotée, qui remédie à la trop grande siccité des fourrages naturels; les especes appartenant aux Papilionacées et Cosalpiniées sont plus rares.

\section{Genre Abrus}

En Afrique tropicale, dans l'Inde, en Australie, Abrus precatorius est une liane bien connue par sa graine, qui sert aux empoisonnements criminels. Dans les buissons auxquels elle se mele, les feuilles sont très recherchées, au Sénégal, par les moutons 
et les chèvres; à Madagascar, on considère qu'elle peut les intoxiquer.

\section{Genre Acacia}

Une place spéciale doit être réservée aux Acacia, dans cette étude sur les arbres et arbustes fourragers. I en existe au moins 400 espèces, réparties dans les régions tropicales et subtropicales des deux mondes; elles y constituent souvent la flore dominante des « steppes à Mimosées ». Leur's utilisations sont nombreuses : l'écorce peut être fibreuse et tannifère; les gousses renferment un tanin utilisé chez certaines espèces; beaucoup, par transformation du contenu amylacé des cellules et de la membrane, donnent à la suite de piqûres d'insectes ou simplement par exsudation naturelle, une gomme dont la plus connue est la « gomme arabique 》. Enfin et c'est là le point de vue qui nous intéresse, beaucoup jouent un rôle important dans l'alimentation des animaux.

Les Acacia ont des arbres ou arbustes à tiges et rameaux inermea ou portant des aiguillons; les rameaux abortifs sont parfois transiormes en épirtes. Le limbe des fevilles est clivise on nombreuses falioles, ou bien avorté, auquel cas le pétiole s'applatit en une lame membraneuse (phylfode). Le日 fleurs, petites, sont généralement nombreuaes, disposées en capitules, ou en épis, ou encore en grappes. Le fruit est une gousse ovale ou oblonque, droite, arquée ou contournée. Ce sont les feuilles les fleurs et les fruits qui sont conoonmes par les animaux, et la vanété présentée par ces trojs éléments assure la diversité dans la valour fourragère des espèces.

Ainsi que le fait remarquer $A$. Chevalier, les Acacia sont d'une grande importance dans les régions subdésertiques où ils abondent, et cela non seulement comme aliment, mais parce que, fixant le sol, ils évitent l'extension du désert et que, possédant des feuilles très fines, ils ne demandent pas beaucoup d'eau au sous-sol. Cela explique la nécessité d'éviter leur dirninution, voire leur disparition, et aussi la grande vénération dont ils sont l'objet de la part des Bédouins, qui disent d'eux qu'ils entourent les frontières du Paradis.

Un avanlage des Acacia, c'est que souvent les diverses espèces d'une région ne lleurissent pas en meme lermps, en sorte qu'ils donnent des gousses à des périodes diflérentes; certains aussi sont en feuilles à une période diff́́rente des autres. Les animaux les consomment de façons diverses. En páturant dans les zones arbustives, ils brautent ce qui est à leur porté el les gouses tombées a terre; la chérre gurtout tire parti an maximum des paturages d'epineux; dans les trouppeaux mixtes de moutons et do ahevres des régions abheliennes, elle est à ce point de vue une excellente éducatrice pour le mouton. Quand les Itracta cont phus hauts, les bergers coupent les grosses branches, voire l'arbre entier, précédant le troupeau avec une hachette el une gaule; c'est un spectacle curieux que de voir accourir le troupeau au bruit de la gaule et de la hache. Les animaux importés sont, de ce point de vue, vite éducrués par les autreo.

Au Soudn anglo-égyptien, les bergers respectent les feuilles et les petites branches des Actcia (A. torilits, A. spirocerpa) quand ils gaulent les leurs et les goussos pour leurs moutons. En d'autre rágions, où les Acacia sont importants, les coutumes des tribus intercisent remplot des gaules.
On peut aussi récolter les gousses pour les distribuer; toutes n'ont pas la même valeur; les meilleures sont les plus tendres et par suite les plus alibiles. A ce point de vue, la première place revient, en Afnque, à $A$. tortilis ( = fasciculata) et $A$. Seyal. Dans les gousses vertes, la graine est facilement digestible, mais il n'en est pas de même pour les gousses mûres de certaines espèces dont les graines traversent le tube digeslif sans être influencées ; elles assurent d'ailleurs de cette façon la dissérnination de l'espèce.

Les Acacia, de façon générale, ne sont pas dangereux. Cependant, une liane de l'Inde, A. rugala, renferme une saponine. Un certain nombre peuvent être cyanogénétiques, en général pas assez pour causer des accidents graves. C'est le cas pour A. glaucescens, $A$. Cheelii, $A$. doratoxylon et A. Cunninghamii, d'Australie, et $A$. giraffa, A. lasiopetala, $A$. Jitakumensis, A. robusta, $A$. stolonefera, d'Afrique du Sud. A. pennata, d'Afrique occidentale, est douteux.

Parmi les nombreuses espèces qui peuplent les Iégions chaudes, il en est qui sont plus ou moins localisées à une région ou à une zone climatique; d'autres ont une aire beaucoup plus étendue et se rencontrent aussi bien en Afrique qu'en Asie. Nous - les étudions par régions.

Afrique occidentale et équatoriale. - D'après A. Chevalier, les Acacia les plus répandus en Afrique occidentale française sont $: A$. fasciculata, $A$. siebe riana, A. gummifera, A. scorpioides, A. arabica (avec les variétés nilotica, pubescens et adstringens), A. Adansonii, A. seyal, A. albida, A. campylacantha, A. mellifera, $A$. senegal, $A$. ataxacantha et $A$. pennata.

$A$. Chevalier les répartit en quatre groupes principaux : $1^{\circ}$ les especes propres au Sahara : A. fasciculata ( $A$. tortilis) et $A$. gummifera, localisées là où elles peuvent rencontrer encore une quantité d'eau assez abondante. Ce ne sont pas à proprement parler des espèces désertiques, mais des vestiges d'une végétation subsistant du temps où le Sahara était mieux arrosé et parcouru par des fleuves. On les trouve dans les oueds ou sur le flane des montagnes, au Maroc, dans le sud algérien et tunisien. On trouve $A$. scorpioides (= arabica) autour des oasis du nord, où il a sans doute été planté; $2^{\circ}$ les espèces de la steppe à Mimosées. Elles sont nombreuses et composent en grande partie cette steppe : $A$. senegal ( $A$. verek), $A$. seyal, $A$. fasciculata, A. albida, A. arabica, A. ataxantha (liane épineuse). fux abords des mares et marigots, dominent souvent $A$. scorpiojdes et $A$. seyal; $3^{\circ}$ les espèces de la brousse soudanaise, de la " forêt parc ". Peu à peu, les espèces précédentes disparaissent pour céder la place, au nord, a $A$. sieberiana et A. albida et 
- espèces plus rares - à $A$. rehmanniana et A. samoryana; sur les bords du Niger moyen : $A$. kirki et, plus au sud, dans le Soudan méridional, la brousse guinéenne, $A$. campylacantha; 40 les especes de la grande forêt, qui ne sont plus arbustives, mais sarmenteuses : A. pennata, $A$, ataxacantha.

A. raddiana ( $=$ A. fasciculata $=$ A. tortilis), dans les régions sahéliennes sablonneuses, constitue de beaux peuplements (arbre de 8 à 10 mètres, à fleurs en boules blanches); les gousses, en forme de faucilles, tombent avant complète dessiccation; ou bien les bergers les gaulent, comme ils coupent les branches. Les gousseg contiennent $20 \%$ de matières saccharifiables.

A. l'inverse du précédent, $A$. senegal, ou vereck, le gommier, est surtout intéressant par ses branches, que les bergers coupents ; les gousses sont appréclées aussi des moutons et chèvres.

Dans les régions plus fraitches, on trouve au bord des mares $A$. seyral dont les nombreuses fleurs en boules, jaune vif, tombent prématurément à terre au début de l'année et forment un véritable tapis; les moutons en sont très friands. Les gousses sont également appréciées.

A. arabica (gonakjé) donne des gousses nummulaires que recherchent les petits ruminants, mais qui sont très riches en tanin. Leur composition moyenne est : protéines, 12; graisse, 2; hydrates de carbone, 5,5 .

$A$. chariensis est une espéce voisine d'A. seyal, séparée de celte dernière par $A$. Chevalier; elle est spéciale au Chari et se rencontrerait aussi en Haute-Volta,

Une variété d'A. seyal (A. seyal var. fistula), à écorce blanchâtre, est dénornmée au Soudan égyptien usoffar \# c'est-à-dire siffleur; c'est que les épines sont attaquées à la base par un insecte qui provoque une hypertrophie considérable des tissus. Quand l'insecte est échappé, il reste une cavité dans laquelle le vent, en s'engouffrant, produit un sifflement caractéristique.

Les gousses d'Acacia (= Faidherbia) albida, larges et épaisses, sont parmi les rares fruits que les indigènes du Sénégal ramassent pour leurs animaux. Pour Chevalier (1934) c'est un fourrage précieux. II retient les terres et fixe l'azote dans le sol. Comme 'arbre est dénudé en saison des pluies et qu'il l'empêche pas la lumiere d'arriver aux cultures serbacées, il est respecté par les cultivateurs noirs. Iussi, au Sénégal et au Soudan, forme-t-il de vériables vergers autour des villages. Les gousses et les branches sont mangées avidement par le bétail.

Au Sénégal, nous avons distribué des goussea pendant deux mois à des bovins; c'est un bon aliment, assez sucré, mais dont il faut eviter une consommation trop prolongée, car il est assez riche en tanin (nous avons trouvé environ $5 \%$ au Sénégal). Les indigènes attribuent à ces gousses des propriétés galactogènes.

A. sieberiana est aussi untéressant, en ce que les feuilles subsistent longtemps pendant la saison sèche; cela pousse les pasteurs à couper, souvent de façon excessive, les branches qu'ils distribuent.

A. loeta est très proche d'A. senegal, avec lequel on le confond souvent; il a les mêmes qualités fourragères.

A. stenocarpa vit dans les bas-fonds humides du Sahel, où il peut former des peuplements denses qui assurent la pâture des moutons.

On trouve aussi notamment en Haute-Côte-d'Ivoire, $A$. pennata et $A$. ataxacantha, qui se présentent comme des lianes; plus au Sud, $A$. campylacantha, A. rehmanniana, dans la zone humide, surtout en ce qui concerne la première espèce. Dans la HauteVolta, A. mellifera, A. samoryana, A. macrostachya. La dermère espece est un arbuste sarmenteux qui se développe beaucoup dans les terrains de culture.

Afrique du Sud. - La variété est grande également. La répartition n'est pas la même selon les régions. Dans le Karoo, on trouve $A$. giraffe, A. robusta, A. Litakunensis comme espèces principales. Dans les régions autres que le Karoo, les espèces qui sont broutées sont : $A$. giraffae, $A$. arabica var. kraussiana, A. robusta, $A$. detinens, $A$. calfra, $A$. litakunensis (spirocarpior̈des). A. Karroo, A. Galpini, A. albida, A. Benthamii, A. Woodii. Pami ces espèces (Henrici, 1947), A. Jitakunensis, A. giraffe. A. Benthamii et A, lasiopetala sont importantes par leurs gousses, pas par leurs feuilles. Les graines sont riches en proteine. Les gousses d'A. litakunensis notamment, sont mangées avidement par les moutons quand elles sont pulvérisées; de même celles d'A. giraffe; les enveloppes sont dures et pauvres, sauf en matières minérales. Les gousses d'A. Karroo et $A$. caoffra, petites, sont peu recherchées, mais leur fevillage, surtout les jeunes pousses, le sont. Quant à $A$. detinens alors que, dans le nord du Transvaal, il est considéré comme trop épineux pour être intéressant, dans les régions pauvres du nord de la province du Cap, les feuilles, fleurs et fruits sont mangés. Les gousses, longues et fines d'A. horrida le sont aussi quand elles tombent à terre. Parmi les nombreuses autres espèces qui peuvent être broutées, il en est dont les gousses peuvent colorer 
le lait; d'autres ( $A$. giraffae) dont les feuilles peuvent renfermer de l'acide cyanhydrique (Steyn, 1943).

Afrique du Nard, Sahara. - Nous retrouvons dans l'Afrique du Nord françalse des especes de la zone sahélienne, communes aussi au Sahara, et des espèces de la region méditerranéenne. Les principales sont : $A$. tortihs, avec des formes et des aspects assez variables selon le terrain, $A$. gummifera du sud marocain, ressemblant beaucoup à l'espèce précédente; $A$. arabica; diverses autres espèces plus ou moins proches d'A. arabica $:$ A. tomentosa, $A$. indica, $A$. mellifera, $A$, orfota, $A$, tubica.

En Somalie, il est des régions où les Acacja dominent parmi la flore arbustive. Deux espèces importantes sont $A$. Bussei, dans I'est de la Somalie britannique; et $A$. thebaica, des régions humides de l'ouest; cette dernière espèce se montre particulièrement résistante aux mauvais traitements des bergers.

Au Soudan anglo-égyptien, nous retrouvons A. albida, à côté d'A. ehrenbergiana, A. mellifera, A. spirocarpa, A. tortilis, $A$. verek, Ce sont A. ehrenbergiana (= A. flava) et A. spirocarpa qui dominent, $A$. tortilis se rencontrant dans les régions moins désertiques. Les gousses de ces deux espèces ont la réputation d'engraisser les moutons sạns qu'on lour donne autre chose, alors que les gousses des autres especces leur permettraient seulement de s'entretenir. A. spirocarpa donne des fleurs pendant une longue période, de janvier à juillet, ce qui fait que pendant la saison sèche il offre toujours des fleurs ou des feuilles. Comme $A$. tortilis fleurit en novermbre, une longue période se trouve ainsi assurée par ces deux especes. En région de savane, on trouve A. seyal, A. mellifera, A. verek.

Africue orientale. - L'Afrique orientale faisant la transition entre l'Afrique du Nord et l'Afrique du Sud, mous trouvons les espéces des deux récions. Dans la zone désertique du Kenya, c'est $A$. mellifera qui domine; dans l'Ouganda, on a recommandé la multiplication d'A. albida en certaines régions menacées par l'érosion. On rencontre aussi, en se rapprochant du sud, $A$. Woodi.

Proche Orient. - Dans le bassin méditerranéen, nous trouvons les especess d'Afrique du Nord, d'ailleurs souvent asiatiques; $A$. arabica, est la plus frécuente; on rencontre aussi $A$. modesta, $A$. indica, A. mellifera.

Inde, Ceylan. - Dans certaines régions de l'Inde, les Acacia sont les arbres fourragers les plus importants. On trouve $A$. arabica, qui domine, $A$. modesta, A. catechu. Dans la province de Madras, A. leucophloea ( $=A$, alba) est très apprécié, ses gousses ayant la réputation de donner un poil brillant. Dans le Punjab, les Acacia dominent, et A. arabica est, là aussi, l'espèce la plus importante. Les feuilles et les grousses sont mangées par tout le bétail. On le retrouve dans le Sind, où il abonde; en avril, alors que les autres fourrages sont rares, ses gousses fournissent un bon appoint; pendant la saison froide. les feuilles sont une excellente nournture, mais on doit réglementer leur coupe, car on a besoin des graines, en grosses quantités, pour ensemencer chaque année pour le reboisement de certanes zones. On réglemente aussi le broutage des Acacia en interdisant aux moutons, aux chevres et aux chaneaux une certaine proportion des pâturages.

Australie. - Au Queensland et dans le territoire du nord, Ies Acacia constituent la formation qu'on appelle le « scrub $n$; les espèces principales étant $A$. aneura, A. cambagei et $A$. harpophylla, arbres de 15 à 20 pieds; c'est la première espece qui est la plus inportante. On rencontre aussi $A$. homejophylla, et $A$. farnesiana, bonnes espèces fourragères mais envahissantes. $A$. excelsa a les mêmes qualités. C.-T. White (1947) donne lés renseignements suivants sur les Acacia du Queensland:

A. aneura est le principal arbre fourrager de l'Ouest, avec des variétés selon les sols;

A. arabica qui est en certaines régions considérée comme une plante envahissante, est un bon appoint par ses gousses, pour les bovins, les moutons et les chèvres;

A. farnesiana, est considéré comme une excellente nourriture pour les moutons (feuilles et gousses vertes); en certaines régions de l'est, il est moins recherché;

A. implexa et $A$. Maidenii, tous deux ì feuilles larges, sont de bonnes espèces fourragères de la zone côtière;

A. pendula, très répandu dans l'ouest, est un excellent appoint en saison sèche.

En Nouvelles-Galles du Sud, on retrouve les mêmes espèces, avec en plus (R.-H. Änderson, 1947):

A. Cheelii, bon fourrage, mais dont les feuilles peuvent contenir de l'acide cyanhydrique:

A. excelsa, couramment mangé, mais qui peut causer de l'indigestion s'il est pris en grande quantité :

A. Loderi, modérément utile;

A. Oswaldii, dont les feuilles et les gousses sont peu recherchées:

A. salicina, de faible valeur.

Dans l'État de Victoria (Colin G. Webb, 1947) les Acacia sont plus rares. Seul est cité $A$. victoriae, de valeur douteuse.

Dans l'Australie du sud, les formations d'Acacia 
ne se rencontrent qu'en des zones limitées : $A$. aneura, A. cambagei, A. victoriae; cette dernière espèce y est considérée comme importante au point de vue alimentaire. On trouve aussi $A$. linophylla, A. salicina, A. Sowdenii, $A$. rigens.

Dans l'Australie de l'ouest, dont une bonne partie jouit du climat tropical, les Acacia sont représentés par un grand nombre d'espèces (il y a, en Rustralie, 320 espèces indigènes d'Aczcia). On les réunit en trois groupes (G.-R.-W. Meadly, 1947) : celui d'A. aneura (" mulga »); celui d'A. Grasbyi et celui d'A. genistoìdes et A. tetragonophylla, qui se distinguent par l'aspect de leurs phyllodes. Selon les espèces, c'est le feuillage ou les gousses qui présentent le plus d'intérêt; dans le premier cas rentre $A$. aneura; dans le deuxième, $A$. Innophylla, A. brachystachya. A. tetragonophylla est une bonne espèce, dont les feulles et les gousses sont mangées.

Amérique du Sud. - Dans les régions à Acacia de l'Amérique du Sud, les espèces suivantes sont fourragères :

A. arabica (Brésil);

A. aroma (Argentine), pousses, feuilles sèches, gousses:

A. cavena (Brésil), pousses et feuilles en été, surtout par les moutons:

A. cebil (Argentine), feulles tombées en hiver, pour les bovins:

A. cymbispina (Mexique), les fruits sont mangés par les chevaux et leg ânes:

A. macracantha (Venezuela), les gousses sont bonnes pour les bovins:

A. moniliformis (Argentine), fruits très recherchés du bétail.

Amérique du Nord. - Il est aussi des zones plus u moins arides et chaudes d'Amérique du Nord où ss Acacia tiennent une place plus ou moins imporunte. C'est le cas pour le Texas ( $A$. angustissima, .. rcemeriana) mais, de façon générale, ils sont d'une ible proportion dans la flore arbustive.

\section{Genre Aeschynomene}

Des espèces brésiliennes, Aeschynomene falcata, , marginata, sont recommandées comme substituts $\Rightarrow$ la luzerne. $A$. americana et $A$. sensitiva sont falement de bonnes espèces.

\section{Genre Afzelia}

Afzelia africana est un arbre d'Afrique occidentale; I feuillage, qui apparait aux mois de janvier, vier, est succulent à ce moment, alors qu'il y a in d'herbe; les bovins le recherchent volontiers. ns certaines régions, on distribue les feuilles au rc. Les graines sont toxiques.

\section{Genre Albizzia}

Comme les Acacia, les Albizzia se rencontrent en Afrique, en Asie, en Australie.

L'espèce la plus répandue est $A$. lebbek, qu'on trouve en diverses régions d'Afrique et d'Asle : Afrique occidentale, Soudan anglo-égyptien, Afruque du Nord, Rhodésie, Indes anglaises, Ceylan, etc. Dans les provinces centrales de l'Inde, il est considéré comme un bon fourrage; de même aux Indes occidentales, où il est recommandé pour les "pâturages à protéine ».

A. lebbek est cultivé en diverses régions. Normalement, il perd ses feuilles en saison sèche; quand on le cultive, comme à Cuba, en plantation serrée (50 $\mathrm{cm} \times 50 \mathrm{~cm}$ ), et qu'on coupe deux ou plusieurs fols par an, on obtient une sorte de prairie et il $n^{\prime} y$ a pas de repos; les feuilles ne tombent pas.

A. Chevaljeri est un arbuste non épineux du Sahel, dont les feuilles et les gousses sont recherchées des numinants; $A$. sassa est moins appréciee, A. zygia est une espèce soudanaise.

A. stipulata, de l'Inde, est considéré comme un bon aliment; on l'utilise comme plante de couverture au lieu de Leucœena glauca, dans les plantations de café.

Toujours dans l'Inde, plusieurs espèces fournissent un feuillage qui est mangé par les bovins, les moutons, les chevres : $A$. amara, $A$. marginata, A. odoratissima, A. procera. $A$. amara est particulièrement appétée; dans la province de Madras, if est une race bovine qui se nourrit à peu près exclusivement de ses feuilles et pousses, même quand il y a de l'herbe.

En Amérique du Sud (Argentine) A. lophante est mangé par le bétail.

En Australie, A. basaltica est particulièrement abondant dans certaines zones sablonneuses et est considéré cornme un bon fourrage. De même A. tharveys en Afrique orientale.

Parmi ces diverses espèces, il en est qui pourraient être dangereuses parce qu'elles contiennent de la saponine: c'est le cas d' $A$. amara, dont les feuilles se vendent dans l'Inde comme substitut du savon; de même, $A$. tophanta, $A$. stipulata, $A$. ferruginea qui est employé en Gold Coast pour les lavages, Ia saponine n'agissant guère par la voie digestive, les accidents sont rares; cependant on en aurait observé avec les pousses jeunes d'A. stipulata. $A$. ferruginea serait, d'autre part, dangereux par un glucoside, la moussénine. I existe aussi en Abyssinie.

\section{Genre Alhagi}

Un buisson à petites feuilles et longues épines, Alhagi camelorum, est très répandu dans les zones désertiques des rives de l'Atlantique au nord de 
l'Asie; il ne convient qu'au chameau, et entre pour bonne part dans son alimentation; ainsi, dans le Baluchistar, les chameaux employés au labour le consomment largement. On le retrouve dans les régions désertiques et subdésertiques de l'Asie. Les feuilles ont la composition suivante : protéine brute, 13,6; extrait éthéré, 4,9; cellulose, 10,1; extractif non azoté, 5,3 .

\section{Genre Ammodendron}

Ammodendron Canollyi est un arbre ou arbuste du Turkestan dont le feuillage est surtout recherché des chameaux.

\section{Genre AnthyIIIs}

Anthyllis henoniana est une bonne espèce, mangée par tous les herbivores, dans les plaines sèches de Libye, Tripolitaine. Les Sahariens l'accusent de causer une paralysie épizootique.

\section{Genre Aspalathus}

Diverses espèces de ce genre sont considérées comme les meilleurs buissons fourragers dans la province du Cap : $A$. virgata, $A$. lepida, $A$. acuminata, A. suffruticosa.

\section{Genre Astragalus}

De très nombreuses espèces asiatiques do ce genre constituent des plantes des régions désertiques ou semi-désertiques; elles fournissent un fourrage moyren. C'est le cas pour Astragalus spinosus, qui convient surtout au chameau. On a recommandé leur multiplication en association avec des Graminées, dans les déserts de l'U.R.S.S.

A. arsatus, en Arabie, en Afrique du Nord, ne peut être donnée aux animaux qu'après avoir été passée au feu pour braler et attendrir la pointe des aiguilles, On la vend ainsi sur les marchés. $A$. bœiticus est également une espèce africaine, qui est parfois récoltée: $A$. proluxus est une espèce d'Afrique du Nord (Cyrénaique, Sahara) qui peut ètre toxique. Plusieurs espèces d'Amérique du Sud sont fourragères : $A$. Bergii, A. unifultus, $A$. atuelli, $A$. chilensis, toutes recherchées du bétail, mais toutes considérées comme toxiques. On sait que de nombreuses especes. d'Amérique du Nord causent la " loco disease $\mathrm{b}$.

\section{Genre Bawhinja}

Dans ce genre, qui appartient aux Cosalpiniées, divers arbres et arbustes sont intéressants. Bauhinia rufescens est un arbuste de la zone sahélienne de l'Afrique occidentale. Dans cette zone, il constitue, nous semble-t-il, le meilleur arbuste fourrager, tant par ses feuilles que par ses gousses (celles-ci a l'état vert seulement). Sa présence dans la flore arbustive d'une région augmente de beaucoup la valeur du pâturage et, en association avec les petites graminées qui profitent de son abri, il peut suffire à l'alimentation de 4 à 5 moutons ou chevres à l'hectare. Malheureusement, il est en voie de disparition dans certaines régions où les bergers le mutilent.

B. reticulata, de I'ouest africain est intéressant surtout par ses gousses.

$B$. Thonningii souvent donné comme synonyme du précédent est également africain. On le trouve en Afrique occidentale, orientale et mérioionale; il est très recherché des divers ruminants; en Afrique du Sud, on estime qu'il suffit à maintenir les moutons en bon état. En Nigeria, on recueille les feuilles et les jeunes pousses pour les distribuer aux moutons et chevres qui demeurent au parc pendant la saison des cultures, quand l'herbe est insuffisamment poussée. Les gousses sont mangées avidement par tout le bétail.

B. petersiana, également africain, est considéré comme un bon fourrage en Afrique orientale. De même B. forficata, du Brésil.

Diverses espèces existent dans les Indes : $B$. purpurea, B. racemosa, B. Vahlii, B. variegata, B. malabarica. Ce sont de bonnes espèces, surtout $B$. malabarica, qu'on trouve aussi dans l'Indochine, les Philippines. D'après Poilane, elle pourrait être appelée à jouer un rôle important dans les pays secs, par ses feuilles et ses gousses: les buffles et les bceufs en font une grosse consommation. B. malabarica a en outre l'avantage de porter fruits à la période de disette fourragère et de donner une grosse quantité de gousses. Cependant, l'analyse révèle une faible quantuté de sucre $(5 \%)$ et pas d'amidon.

En Australie, on rencontre B. Carronii, mangé surtout par les moutons, $B$. Cunninghamii, $B$. esculenta, B. Hookeri. Les Gousses de B. esculenta sont riches en protéine, matière grasse et hydrates de carbone.

\section{Genre Bowdichia}

Dans les zones arides du Brésil, Bowdichia virgilioldes a une bonne valeur fourragere.

\section{Genre Bradbarya}

Des espèces de ce genre constituent, au Brésil, au Salvador, des plantes grimpantes que mange le bétail (Bradburya sagittata).

\section{Genre Caesalpinia}

Plusieurs espèces de ce genre, Caesalpinia bracteosa, C. corymbosa, C. ferrea var. cearensis, C. pyramidalis, sont des arbres des régions arides 
du Brésil intéressants; C. bracteasa en particulier, dont les pousses sont les premières à sortir apres une longue période de sécheresse. Le bétail les dévore alors littéralement. Les fruits de C. melanocarpa sont mangés par les chèvres.

\section{Genre Calliandra}

Sur les terrains pierreux des steppes du Mexique, on rencontre Calliandra grandiflora, espèce herbacée ou sous-buissonneuse que le bétail mange volontiers. Dans les zones chaudes et andes d'Amérique du Nord (Texas, Arizona, Mexique), C. eriophylla est un buisson bas dénommé « faux Prosopis » dont les feuilles et gousses auraient la valeur de la luzerne.

\section{Genre Calparnia}

Les feuilles de Calpurnia subdecandra, d'Afrique du Sud, sont estimées du bétail.

\section{Genre Calycotome}

Calycotome intermedia, qu'on trouve en Tripo litaine dans les terrains rocheux et compacts, peut être non seulement pâturée, mais cultivée en rotation.

\section{Genre Carmichaelia}

Une vingtaine d'espèces de ce genre sont localisées à la Nouvelle-Zélande. Elles sont pratiquement sans feuilles: les pousses sont mangées par les bovins, les chevaux, les moutons. Les principales espèces sont Carmichælia Munroi, C. Pretrei, C. subulata, C. odorata, C. compacta.

\section{Genre Cassia}

Ce genre comprend de nombreuses espèces dont certaines sont utilisées dans la pharmacopée: d'autres sont dangereuses; un certain nombre sont fourragères.

En Afrique occidentale, Cassa sieberiana a de longues gousses rectilignes très recherchées des animaux; les indigènes les récoltent pour les distribuer aux vaches laitières (Guinée, Soudan). C. mimosoides est mangée par tous les herbivores; en Nigeria, C. trotschyana est surtout une plante à chamealu.

Dans l'Inde, les feuilles de C. fistula, C. Siamea, C. nictitans, sont mangées par les bovins, les moutons, les chèvres.

En Australie, C. artemisoides, C. Sturtii, C. eremophila, bien que mangées, sont de valeur incertaine. En Amérique, C. romeriana, du Texas, est mangée par le bétail (feuilles et gousses).
Signalons aussi $C$. acutifolia, d'Afrique du Nord et d'Arabie, dont le chameau recherche les feuilles; les effets purgatifs peuvent être dangereux; même remarque pour $C$. obovata, des mêmes régions.

Dans les régions arides et chaudes de l'Amérique du Sud, plusieurs espèces sont mangées : C. acuta (Chyli) par tous les animaux sauf le cheval ; C. crassiramea (Argentine) par les chèves; $C$. ferruginea; C. fistula; C. multijuga; C. rotundifolia, qui donnerait un bon foin; C. tora, recommandé pour l'ensilage ou le fanage. A Cuba, on cultive C. siamea comme plante de couverture.

\section{Genre Ceratonia}

Le caroubier, Ceratonia siliqua est maintemant répandu en diverses régions; on $\mathrm{l}^{\top} \mathrm{a}$ introduit en Australie, en Nouvelle-Zélande; on le cultive en Afrique du Nord, en Afrique orientale. C'est la production des gousses qu'on recherche.

\section{Genre Chamæcrista}

Chamæcrista flexuosa est une Légumineuse herbacée semi-couchée, des savannes guyanaises, que le bétail mange volontiers.

\section{Gence Clitoria}

Plusieurs espèces volubiles de ce genre sont fourragères, mais de faible appoint : Clitoria sp., souvent utilisée comme plante ornementale en Afrique occidentale; $\mathrm{Cl}$. ternatea, utilisée comme plante de couverture aux Philippines, est une espèce d'Afrique orientale (Tanganyika).

\section{Genre Corallospartium}

Le genre comprend une seule espece, Corallospartium crassicaúle, arbrisseau buissonneux de Nouvelle-Zélande, à branches assez dures que mangent cependant les bovins et les moutons.

\section{Genre Copaifera}

Copaifera mopane est répandu en certaines régions d'Afrique du Sud; cet arbre est recherché du bétail: ses feuilles sont riches en protéine et phosphore (Henrici) et, bien qu'ayant une forte odeur de térébenthine, ne transmettent pas cette odeur au lait. Elles ne peuvent cependant suffire à l'alimentation du bétail (Pole Evans, 1945).

\section{Genre Coragana}

Coragana frutescens, C. pygmea, du Turkestan, sont mangèes par le chameau. 


\section{Genre Cordeauxia}

Cordeauxia edulis est une Légumineuse assez répandue en Somalie; la graine que mangent les indigènes est riche en graisse et sucre, mais plus pauvre en protéine que les autres graines de Légumineuses. La composition est la suivante (Fabiani, 1940) :

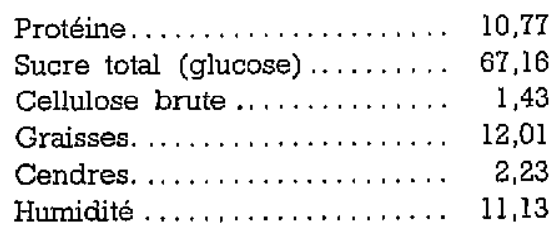

La graine est pauvre en carotène et en $\mathrm{Ca}$ (Greenway et Raymond, 1947).

\section{Genre Cratylia}

Dans les zones arides du Brésil, Cratylia mollis est une bonne plante fourragère.

\section{Genre Crotajaria}

Diverses espèces de ce genre sont cultivées. Parmi les espèces spontanées qui peuvent être mangées, citons Crotalaria arenaria du sud saharien, C. senegalensis, de la zone soudanaise, qu'on retrouve en Afrique orientale, C. quartiniana, C. saxatilis, également africaines. De nombreuses espèces sont mangées; beaucoup d'autres sont délaissées.

\section{Genre Cyamopsis}

Cyamopsis psoraloides est mangée (Indes).

\section{Genre Cytisus}

Plusieurs espèces sont des arbustes fourragers, notamment Cytisus proliferus, le tagasaste, qui a été preconisé en Afrique du Nord; on le cultive en Australie, sous le nom de "luzerne arborescente", comme haie, brise-vent; dans certaines régions de I'Extrême-Orient on l'utilise comme arbuste fourrager.

Il $\mathrm{y}$ a trois variétés; la variété palmensis est le yrai tagasaste.

Aux Canaries, il servirait à l'alimentation du cheval et du mouton. En Australie, les essais ont montré que les animaux ne le consomment pas volontiers. On le cultive aussi-au Chili.

La composition du foin est la suivante :

Eau ..................... 11

Cendres................. 6,5

Matières azotées ............ 12,81

Cellulose ................ 16

Hydrates de carbone........ 51,89

Matières grasses........... 1,70
On sait que divers Cytisus peuvent causer des troubles. Ce ne parait pas être le cas pour C. proliferus. Cependant, on aurait observé des accidents chez des chevaux.

En Australie, on a acclimaté d'autres espèces : C. scoparius, C. stenopetalus, C. monspessulanus. C. scoparius convient surtout aux moutons, alors que C. monspessulanus est mangé également par les bovins.

\section{Genre Dalbergia}

Dalbergia sissoo, $D$. Jatifolra, tous deux de l'Inde, ont des feuilles comestibles. De même $D$. melanoxylon, de l'Afrique occidentale et orientale.

\section{Genre Dalea}

Ce genre comprend, en Amérique du Sud, des espèces herbacées poussant dans les régions semiarides du Mexique : Dalea citriodora, D. Puerryi, D. polygonoïdes.

\section{Genre Daniella}

Daniella oliveri, d'Afrique occidentale, a des feuilles que mangent grands et petits ruminants.

\section{Genre Desmanthys}

Des herbes de ce genre constituent de bons fourrages : Desmanthus virgatus (Hawaī), D. depressus (Uruguay). Des espèces du genre sont cultivées en d'autres régions.

\section{Genre Desmodium}

Ce genre renferme des arbustes, des arbres et aussi des espèces buissonnantes utilisées comme plantes de couverture.

Desmodium umbellatum, du Queensland, y est considéré comme un excellent fourrage, particulièrement pour les chevaux; de même $D$, varians. D. pulchellum, arbre de l'Inde, est aussi considéré comrne un bon fourrage. Plusieurs especes existent en Afrique occidentale : $D$, adscendens, $D$. gangeticum.

\section{Genre Dichrostachys}

Dichrostachys glomerata, du nord du Transvaal, est un arbuste dont les herbivores mangent les feuilles et les gousses. On le retrouve en Afrique occidentale. $D$. nutans, au Soudan égyptien, en Afrique occidentale, a des feuilles qui sont un assez bon fourrage, ainsi que les gousses. Ces dernières ont la composition suivante : protéine, 10,8\%; graisses, $1,42 \%$; cellulose, $26,64 \%$ i hydrates de carbone, $55,76 \%$. 


\section{Genre Entada}

Entada sudanica est un arbre de la savane africaine dont les feuilles sont un bon fourrage. On en a même conseillé la culture.

\section{Genre Erinacea}

Erinacea pungens, d'Afrique du Nord, est un " genêt " que mange le chameau.

\section{Genre Erythrina}

Erythrina indica est un arbuste de l'Inde dont les feuilles sont comestibles. Celles $d^{\prime} E$. senegalensis également, ainsi que celles d'E. mulungu, du Brésil, E. Jithosperma (zone méditerranéenne).

\section{Genre Flemingia}

Flemingia faginea, du sud saharien et du Sahel, est mangée par le chameau.

\section{Genre Galactia}

Des plantes herbacées de ce genre constituent de bons fourrages. Galactia gracillima en Uruguay, disparast par le broutage. G. jussicuana, de la savane vénézuélienne, est peu répandue.

\section{Genre Genista}

Plusieurs genêts des zones désertiques on subdésertiques d'Afrique du Nord ont des pousses et des feuilles comestibles. C'est le cas pour Genista saharæ, très prisé, mais parfois considéré comme dangereux; G. capitellata, du Sahara; G. tunetana, de Tripolitaine, sont aussi des arbustes à chameau.

\section{Genre Gleditschia}

A ce genre appartient l'Acacia à miel, Gleditschia triacanthos, qui est cultivé en Afrique du Sud (particulièrement dans le Basutoland), en Amérique du Sud et en Australie. Il réussit bien lả où l'hiver est assez marqué : darns l'État d'Orange, les moutons en apprécient beaucoup les fruits; cependant, quand ces demiers sont tombés à terre, ils s'altèrent rapidement et sont délaissés. Par contre, au Basutoland, le bétail ne les mange jamais (Henric). Les porcs les mangent au Brésil, en Argentine; on les réduit en poudre pour l'alimentation du bétail. La compositon est la suivante : protéine, 23,1 ; hydrate de carbone, 54,2; graisse, 4,6; cellulose, 12.7 (Locke, 1947).

\section{Genre Gliciridia}

Gliciridia sepium, en Amérique du Sud (Venuzuela) est avidement mangée par les bovins à la fin de la saison sèche (feuilles). Elle serait toxique pour les chevaux. On en fait de bonnes haies. Gl. maculata, à Ceylan, a également des feuilles recherchées des bovns.

\section{Genre Glycyrshiza}

La réglisse, Glycyrrhiza glabra, forme en Asie de grandes étendues où pâture le chameau. Dans la République Argentine, le mouton mange Gl. astragalina.

\section{Genre Clycine}

Glycine javanica est une bonne espece (Kenya).

\section{Genre Indigofera}

Les espéces de ce genre forment en général des buissons dont certains sont mangés par le bétail. C'est le cas pour Indigofera pulchella, I. pauciflora, de l'Inde, et $J$. ruspoli, d'Afrique orientale. Ce dernier tient une place importante dans certains paturages de Somalie, du Kenya ainsi que I. tettensis, I. endecaphylla, I. retrollexa. I. semitrijuga, du sud saharien, I. sessiliflora, d'Afrique occidentale, sont également mangées, ainsi que I. pascuorum, I. lespedezjoides, au Venezuela.

\section{Genre Inga}

Dans les zones arides du Brésil. Inga affinis a une bonne valeur fourragère. Au Guatemala, on utilise aussi les graines d'I. paterno, bouillies ou séchées après écrasement.

\section{Genre Lebeckia}

En Afrique du Sud, des arbustes du genre, Lebeckia cinerea, L. spinescens, L. serecia sont d'assez bons arbustes fourragers. $L$. cinerea est recommandé contre l'érosion. Les feuilles de L.: serecia renferment $20 \%$ de protéme.

\section{Genre Lessertia}

Un arbuste buissonnant d'Afrique du Sud, Lessertia depressa, est un excellent fourrage.

\section{Genre Lotonotis}

Lotonotis divaricata et $L$. genuflexa sont des arbustes du Karoo ( $A$ frique du Sud) qui constituent un excellent fourrage. Ils seraient même trop riches et devraient être mélés à une ration plus pauvre (Henrici). La composition des feulles de L. divaritaca est la suivante : eau, 62,9 ; protéine brute, 12,7; extrait éthéré, 4 ; cellulose brute, 34,6; extractif non azoté, 41.4 ; cendres, 8,2 . 
Une autre espèce de l'Afrique australe, L. invoJucrata peut contenir assez d'acide cyanhydrique pour être dangereuse. Il existe aussi des espèces méditerranéennes.

\section{Genre Lotus}

Lotus creticus, espèce spontanée de Libye, Tripolitaine, de la zone méditerranéenne fournit, dans les terrains sablonneux de la zone maritime, une bonne espèce qui se vend sur les marchés. $L$. cytisor̈des est aussi un bon fourrage. L. arabicus, dans la vallée du Nil, peut être cyanogénétique, au début de la vie végétative seulement. Des espèces sont cultivées en diverses régions.

\section{Genre Mimosa}

Mimosa polyanthana, la sensitive du Sénégal, est consommable. De même M. rubicaulis, du Sahara, M. coesalpiniffolia, du Brésil, $M$. fragrans, du Texas, et une espèce de Rhodésie, $M$. invisa est distribuée aux bovidés, aux Indes néerlandaises; elle pourrait causer des accidents.

\section{Genre Ononis}

Ononis falcata est vendue sur les marchés de Libye, Tripolitaine.

\section{Genre Ormocarpum}

Le betail mange volontiers Ormocarpum Kirkii, d'Afrique orientale.

\section{Genre Ougreinja}

Ougeinia dalbergioídes est un arbre de l'Inde. trés répandu, dont les feuilles sont appréciées.

\section{Genre Oxytropis}

En Asie, en Amerique, des sous-arbrisseaux de ce genre foumissent un bon fourrage. C'est le cas de Oxytropis pilosa; par contre, d'autres espèces peuvent être dangereuses (O. Lamberti).

\section{Genre Parkia}

Parkia africana (= P. biglobosa) est le " néré \# d'Afrique occidentale. La pulpe de ses gousses est utilisée par les indigènes en raison de sa richesse en sucre. Les gousses sont parfois données au bétail. Les graines ont une valeur égale à celle du soja ou de l'arachide. Elles sont riches en vitamine $\mathrm{B}_{\mathfrak{q}}$; elles ne renferment pas d'amidon, mais une huile siccative dans la proportion de $25 \%=$ (Lecoq et Mle Raffy, 1944).

\section{Genxe Parkinsonia}

Parkinsonia aculeata est un arbuste asiatique qui a été introduit en Afrique, en Australie surtout comme arbuste d'ornement, d'ombrage, également comme clôture, dans les régions sablonneuses sèches. Les folioles sont estimées des moutons et des chèvres. Les feuilies vertes et les fleurs ont la composition suivante : protéine brute, 19,3; extrait éthéré, 1,8; cellulose brute, 22.4 ; extractif non azoté, 46.2 ; cendres, 1 .

\section{Genre Piptadenia}

Les arbres de ce genre, dans les zones arides et chaudes d'Amérique du Sud, ont une certaine valeur fourragère : Piptadenia spp., au Brésil, en Argentine; $P$. communis, au Brésil; P. moniliformis, au Brésil; les feuilles de cette dernière espèce pourraient être dangereuses.

\section{Genre Pithecolobizum (Samanea)}

Des arbres de ce genre, probablement d'origine sud-américaine, maintenant africains et asiatiques, ont des feuilles et des gousses comestibles. Ce sont notamment Pithecolobium et $P$, saman. En Indochine, les graines de ce dernier renferment $21, \% 5$ de matières azotées et $49 \%$ de matière grasse; on peut les donner aux bovins; les gousses sont egalement distrubuées; elles renferment $70 \%$ de matières albuminoijdes et $11 \%$ de matière grasse (Rémond et Winter, 1944).

$P$. dulce a été introduit en divers points d' Afrique, notamment au Soudan égyptien; les indigènes l'utilisent comme haie et distribuent les coupes aux animaux. En Gold Coast, où $P$. saman a été introduit d'Amérique du Sud, les gousses constituent un excellent aliment pour les bovins.

P. Iobatum, de l'Inde, a des gousses qui présentent une odeur désagréable. Cependant, les herbivores les mangent parfors, et on pourrait alors observer des troubles dugestifs.

Les espèces sud-américaines sont : $P$. assaremotemo, qui peut être périodiquement toxaque; P. dulce: P. guaricense, P. tortum, du Venezuela: $P$. Lgustrinum, dont les feuilles sont mangées; $P$. mexicanum, dont les corolles sont mangées à terre.

Au Venezuela, les feuilles de $P$. ligustrinum sont considérées comme un excellent fourrage pour les chèvres, qui engraisseraient même avec les feuilles seches.

\section{Genre Prosopis}

Dans ce genre, plusieurs especes se montrent très intéressantes et ont même été multipliées comme arbres fourragers, dans les régions sèches surtaut. C'est ainsi que Prosopis juliflora, originaire proba- 
blement d'Amérique du Sud, a été introduit en de nombreuses régions. Chevalier indique de nombreux symonymes; Minosa juliflora, $P$. cumarrensis, P. dulcis, P. pallida, Acacia falcata. C'est un petit arbre conmun au Mexique (où on le dénomme "mes. quito $n$ ) et dans la partie subtempérée des États-Unis (Californie, Texas). Il a une variété américaine (Texas, Mexique), P. glandulosa. Dans ces régions, il pousse là où la caroubier ne peut venir; dans les zones sablonneuses sèches, il forme parfors de grandes étendues, seul ou mêlé à des Yucca.

De taille trés variable (de buisson à $20 \mathrm{~m}$ ), il a une gousse comprimée qui est un bon alument accepté par les animaux; il est utilisé comme engrais vert; il pousse dans les sols sablonneux secs et a bien réussi en Afrique occidentale, oì il a été largement répandu surtout comme plante d'ombrage; les animaux ne m'en ont pas paru très friands.

La partie la plus intéressante du fruit est la pulpe, qui contient environ $63 \%$ de sucre fermentable. Le fruit comprend $74 \%$ de pulpe. Les autres composants de la pulpe sont : protéines, 11,4; graisse, 4,26; anidon, 8,18 ; cellulose, 4,4 .

P. juliffora a été introduit en Australie, au Soudan anglo-égyptien. On le trouve aussi dans l'Inde, ainsi que $P$. spicigera, dont les feuilles et les gousses sont recherchées des ruminants. Dans les plaines de l'Inde, les Prosopis donnent deux récoltes de gousses par an; leur système radiculaire en fait par ailleurs de bons agents contre l'érosion. $P$. chilensis, dénommé, comme le précédent, «mesquito \%, est recommandé dans les Indes occidentales pour les "pâturages à protéines ", surtout à cause de ses gousses. Plusieurs variétés ont été introduites en Afrique du Sud : $P$. chilensis velutina, $P$. chilensis glandulosa. La variété velutina est particulièrement résistante à la sécheresse (Henrici), à l'égal de P. dulcis ( $=P$. julifflora).

Les gousses de $P$. chilensis, en Afrique du Sud, ont la composition suivante : protéine, 14,7 ; hydrates de carbone, 59,7; graisses, 3; cellulose, 18,3 (Loock, 1947).

Les Prosopis sont très répandus en Amérique du Sud, qu'ils soient indigènes ou introduits $: P$. alba (Argentine, Brésil); P. alpataco (Argentine); P. caldenia (Argentine). P. campestris (Brésil); P. chilensis (Argentine, Mexique, Pérou); $p$. dulcrs (Chili); $P$. julliflora (Brésil, Guatemala, Mexique, Pérou); P. nigra (Argentine); P. santa (Argentine); $P$. ruscifolia (Argentine); $P$. tamarugo (Chuli). Ce sont les fruits qui, en général, sont mangés, parfors les feuilles. On trouve aussi, dans les terrains arides, la pampa d'Ärgentine, $P$. humilis, $P$. striata, P. strumbulifera.

P. africana est un grand arbre des régions nord de l'Afrique occidentale, dont les bovins, moutons el chèvres mangent les feuilles, fines et tendres.

P. stephaniana est fréquent, en Irak, en Kurdistan, où il forme parfois de véritables fourrés; il est volontiers consommé en été.

\section{Genre Psoralea}

Psoralea obtusifolia est un arbuste de l'Afrique du Sud considéré comme excellent mais qui pourrait causer des accidents quand il est fané. Ps. hirta est aussi un bon fourrage. Ps. plicata d'Asie, Arabie, est surtout mangée par le chameau. Une espece australienne est recherchée. Ps. glandulosa, du Chili, est mangée par tous les animaux sauf le cheval. D'autres espèces sont considérées cornme dangereuses.

\section{Genre Pterocarpus}

Plusieurs arbres de ce genre ont de bonnes qualités fourragères. C'est surtout le cas de Pterocarpus erinaceus, assez répandu en Afrique occidentale; les feuilles et les fruts sont mangés, Les fruits forment de grosses grappes dont on peut recueillir des quantités importantes un peu avant la maturité. Les animaux en sont friands, surtout les bovins, la graine étant entourée de grandes ailes membraneuses tendres.

Les feuilles de Pt. marsupium, Pt. santalinus, de l'Inde, sont mangées par les bovins, les moutons et les chèvres.

Pt. rotundifolius est un arbuste du Transvaal dont les feuilles sont mangées par tous les herbivores au printemps, et aussi en hiver quand elles sont tombées.

\section{Genre Retama}

Retama retama est une espèce d'Afrique du Nord, de Tripolitaine dont seules les feuilles sont mangées.

\section{Genre Shotia}

Shotia latifolia et $S$. speciosa sont des arbustes d'Afnque du sud qui sont abondamment broutés.

\section{Genre Sophora}

Ce genre comprend des arbustes, parfois des arbres, très répandus en régions tropicales. Les feuilles de la plupart sont mangées par les animaux; les graines sont souvent toxiques, parce qu'elles contiennent un alcaloïde. Sophora tomentosa est distribuée aux animaux à Madagascar: S. secunzdillora au Texas; le même causerait, au Mexique, des accidents mortels, ainsi que $S$. serica.

\section{Genre Spartikm}

Spartium junceum, le genêt d'Espagne, qu'on trouve dans la zone méditerranéenne, existe aussi 
en Amérique (Pérou, Mexique), Les pousses jeunes sont mangées par les chevaux, les moutons, les chevres, les lamas.

\section{Geinre Stryphnodendron}

Dans les zones arides du Brésil, Stryphnodendron obovalum est un arbre de bonne valeur fourragère.

\section{Genre Sutherlandia}

Deux arbustes buissonnants du Karoo (Afrique du Sud) sont recherchés du mouton au début de la végétation, puis délaussés en raison de leur goût amer. Ce sont Sutherlandia humilis et $S$. microphylla. Les feuilles du premier ont la composition suivante: protéine brute, 22,5; extrait éthéré, 3 ; cellulose brute, 13,4 ; extractif non azoté, 47,8 ; cendres, 13.

\section{Genre Swainsonia}

Des arbustes de ce genre, Swainsonja procumbens, SW, phacoides, sont d'excellentes plantes fourragères en Tasmanie alors que d'autres sont douteuses : Sw, Oliveri, Sw. gregana.

\section{Cenre Swartzia}

Les gousses de Swartzia madagascariensis, qu'on rencontre à Madagascar, en Afrique du Sud, en Afrique occidentale, sont recherchées.

\section{Genre Tamarindus}

Les gousses du Tamarinier, Tamarindus indica, en Asie, en Afrique, sont très goûtées des ruminants.

\section{Genre Tephrosia}

I côté des espèces toxiques du genre, il existe en Afrique occidentale plusieurs Tephrosia qui sont de bons fourrages : T. polystachia, T. purpurea. $r$. candida, connue à la Réunion sous le nom d'indigotier, y est donnée aux vaches, alors qu'elle est considérée comme toxique à l'ile Maurice, T'. incana est une bonne espèce d'Afrique orientale.

\section{Genre Trigonella}

En dehors du fenugrec, Trigonella foenum grœecum, cultivé, on trouve en Afrique du Nord, Sahel, Libye, Tripolitaine, de bonnes espèces spontanées : Tr. anguina, Tr. laciniata.

\section{Genre Viborgia}

Viborgia fusca est un arbrisseau fourrager intéressant d'Afrique du Sud, ainsi que $V$, sericea.

(A suigre) 